\title{
Producción de chile dulce (Capsicum annuиm) en invernadero: efecto de densidad de siembra y poda
}

\section{Sweet pepper (Capsicum annuиm) production under greenhouse conditions: effect of plant density and pruning}

\author{
José Eladio Monge Pérez ${ }^{1}$ \\ Universidad de Costa Rica, Costa Rica
}

\author{
Michelle Loría Coto ${ }^{2}$ \\ Universidad Estatal a Distancia, Costa Rica
}

\begin{abstract}
Resumen. Se evaluó el efecto de dos tipos de poda (española y holandesa) y tres densidades de siembra (2.60; 3.25; y 3.90 plantas $/ \mathrm{m}^{2}$ ) sobre el rendimiento y la calidad del chile dulce cv. FBM-9 cultivado bajo invernadero en Costa Rica. Se obtuvieron más frutos totales por planta, pero de menor peso promedio, cuando se utilizó poda española que cuando se aplicó poda holandesa. Con la poda española, se obtuvo un mayor número total de frutos/m² (95.19) y un mayor rendimiento comercial (66.98 t/ha) que con la poda holandesa (61.45 frutos/ $\mathrm{m}^{2}$ y $45.24 \mathrm{t} / \mathrm{ha}$, respectivamente). La densidad de siembra no afectó el peso promedio de los frutos, pero el número total de frutos por metro cuadrado fue mayor a las densidades de 3.90 y 3.25 plantas $/ \mathrm{m}^{2}$ (83.24 y 82.17 frutos $/ \mathrm{m}^{2}$, respectivamente); el rendimiento total fue mayor a las densidades de 3.90 y 3.25 plantas $/ \mathrm{m}^{2}$ (76.54 y $73.06 \mathrm{t} / \mathrm{ha}$, respectivamente); el rendimiento comercial fue mayor a las densidades de 3.90 y 3.25 plantas $/ \mathrm{m}^{2}$ (62.84 y $57.70 \mathrm{t} / \mathrm{ha}$, respectivamente); y el número total de frutos por planta fue mayor a las densidades de 2.60 y 3.25 plantas $/ \mathrm{m}^{2}$ (26.75 y 25.28 frutos/planta, respectivamente). Los tratamientos que presentaron el mayor rendimiento comercial y el mayor número de frutos totales $/ \mathrm{m}^{2}$ fueron la poda española, tanto a 3.25 plantas $/ \mathrm{m}^{2}$ (71.00 t/ha; y 102.99 frutos $/ \mathrm{m}^{2}$, respectivamente) como a 3.90 plantas $/ \mathrm{m}^{2}$ (73.80 t/ha; y 99.70 frutos $/ \mathrm{m}^{2}$, respectivamente); entre ambas opciones, el uso de la densidad de 3.25 plantas $/ \mathrm{m}^{2}$ representa la mejor desde el punto de vista económico.
\end{abstract}

Palabras clave. Invernadero, calidad, rendimiento, poda, densidad de siembra

\begin{abstract}
The effect of two types of pruning (Spanish and Dutch) and three planting densities (2.60; 3.25; and 3.90 plants $/ \mathrm{m}^{2}$ ) on the yield and quality of sweet pepper cv. FBM-9 grown under greenhouse conditions in Costa Rica, was evaluated. Spanish pruning yielded more total fruits per plant but of lower mean weight than Dutch pruning. Spanish pruning yielded more total fruits $/ \mathrm{m}^{2}$ (95.19) and higher commercial yield (66.98 T/ha) than Dutch pruning (61.45 fruits $/ \mathrm{m}^{2}$ and $45.24 \mathrm{t} / \mathrm{ha}$, respectively). Plant density didn't have effects on the mean fruit weight, but the total number of fruits per square meter was higher at densities of 3.90 and 3.25 plants $/ \mathrm{m}^{2}$ (83.24 and 82.17 fruits $/ \mathrm{m}^{2}$, respectively); the total yield was higher at densities of 3.90 and 3.25 plants $/ \mathrm{m}^{2}$ (76.54 and $73.06 \mathrm{~T} / \mathrm{ha}$, respectively); the commercial yield was higher at densities of 3.90 and 3.25 plants $/ \mathrm{m}^{2}$ (62.84 and $57.70 \mathrm{t} / \mathrm{ha}$, respectively); and the total number of fruits per plant was higher at densities of 2.60 and 3.25 plants $/ \mathrm{m}^{2}$ (26.75 and 25.28 fruits/plant, respectively). Spanish pruning showed the highest commercial yield and the highest total number of fruits $/ \mathrm{m}^{2}$, both at $3.25 \mathrm{plants} / \mathrm{m}^{2}(71.00$ $\mathrm{T} /$ ha; and 102.99 fruits $/ \mathrm{m}^{2}$, respectively) and 3.90 plants $/ \mathrm{m}^{2}$ (73.80 T/ha; and 99.70 fruits $/ \mathrm{m}^{2}$, respectively); between both options, the use of a planting density of 3.25 plants $/ \mathrm{m}^{2}$ represents the best from an economical point of view.
\end{abstract}

Keywords. Greenhouse, quality, yield, pruning, plant density

\footnotetext{
${ }^{1}$ Ingeniero agrónomo, investigador de la Estación Experimental Agrícola Fabio Baudrit Moreno y docente de la Sede de Guanacaste, Universidad de Costa Rica, Costa Rica. Dirección electrónica: melonescr@yahoo.com.mx

${ }^{2}$ Economista agrícola, investigadora y docente de la Escuela de Ciencias Exactas y Naturales, Universidad Estatal a Distancia, Costa Rica. Dirección electrónica: michelle loria@yahoo.com
} 


\section{Introducción}

El chile dulce (Capsicum annuum L.) es una de las principales hortalizas que se cultivan en Costa Rica, mayoritariamente a campo abierto, pero también en invernadero. En un estudio realizado en el año 2003, la mayor parte de los invernaderos del país estaban localizados en la Región Central, y las principales hortalizas que se cultivaban en ellos eran chile dulce y tomate (Marín, s.f.). Entre los años 2008 y 2009, el área total de cultivo protegido de tomate y chile dulce a nivel nacional fue de 116 hectáreas, aunque solo el 35\% de dicha área correspondió a invernaderos (Marín, 2010).

El chile dulce se considera una fuente balanceada de la mayoría de nutrientes esenciales (como calcio, fósforo, potasio e hierro), con alto contenido de vitaminas (particularmente provitamina $A$, vitamina $B$ y vitamina $C$ ), además de importantes antioxidantes, y es rico en flavonoides y fitoquímicos (Maboko, Du Plooy y Chiloane, 2012; Tsegay, Tesfaye, Mohammed, Yirga y Bayleyegn, 2013).

Este cultivo es objeto de mucha atención desde hace algunos años, debido a que se le asocia con la prevención de ciertos tipos de enfermedades cardiovasculares, arterosclerosis, cáncer, hemorragias, el retraso del proceso de envejecimiento, la evitación del colesterol, el mejoramiento de la resistencia física y el aumento del apetito (Tsegay, Tesfaye, Mohammed, Yirga y Bayleyegn, 2013); además, es una hortaliza con bajo contenido de calorías (Sakaldas y Kaynas, 2010).

En el año 2009, las exportaciones de chile dulce de América Central y el Caribe hacia Estados Unidos contabilizaron más de 10500 toneladas métricas (Reho, 2010); en esa actividad se utilizaban únicamente variedades con frutos de forma cuadrada, de colores anaranjado, amarillo y rojo, y se hacía bajo ambientes protegidos, tales como invernaderos, túneles y otros, que permiten el mejoramiento de la calidad del fruto comparado con la producción a campo abierto. En el año 2010, los principales países productores de esta región de acuerdo a su volumen fueron República Dominicana, Honduras, Nicaragua y Guatemala que, en conjunto, representaban en ese momento más de 600 hectáreas de ambientes protegidos (Santos, y otros, 2011). En Costa Rica, la exportación de chile dulce hacia EE. UU. inició en el año 2012 (Salas, 2012; Arias, 2013).

El rendimiento de chile dulce cultivado en invernadero es mayor que a campo abierto; en EE. UU. se estimó que la producción en invernadero varía entre 60 y $260 \mathrm{t} / \mathrm{ha}$, mientras que a campo abierto fue de entre 28.5 y $33.7 \mathrm{t} / \mathrm{ha}$ (Jovivich, VanSickle, Cantliffe y Stoffella, 2005).

La rentabilidad de un cultivo hortícola producido en invernadero depende de la capacidad para obtener un alto rendimiento y una mejor calidad por unidad de área. En chile dulce, una baja densidad de siembra aumenta la producción por planta, pero disminuye la producción por unidad de área (Jovicich, Cantliffe y Hochmuth, 1999; Maniutiu, Sima, Apahidean, Apahidean y Ficior, 2010), debido a que la densidad de siembra afecta la intercepción de la luz por parte del cultivo, y es un factor que puede ser utilizado para mejorar el rendimiento de frutos por unidad de área (Maniutiu, Sima, Apahidean, Apahidean y Ficior, 2010).

El peso (y por ende, el tamaño) del fruto de chile dulce es una variable muy importante, ya que determina el precio del producto, pues los frutos más grandes tienen mayor precio (Jovicich, Cantliffe y Hochmuth, 1999). Una forma de modificar el tamaño de los frutos de chile dulce en invernadero es mediante la poda de tallos (Jovicich, Cantliffe y Hochmuth, 1999); esta práctica mejora la intercepción de luz en el dosel, el cuaje de frutos y su calidad (Jovicich, Cantliffe y Stoffella, 2004). Una forma de 
optimizar la producción comercial de chile dulce por unidad de área es mediante la combinación apropiada de densidad de siembra y sistema de poda (Jovicich, Cantliffe y Stoffella, 2004; Aminifard, Aroiee, Ameri y Fatemi, 2012).

En chile dulce se han establecido dos tipos principales de poda: española y holandesa (Gamayo, 1996). Con la poda española se logra un ahorro del $75 \%$ de los costos de mano de obra, en comparación con la poda holandesa; sin embargo, con la poda holandesa a veces se logra producir frutos de chile dulce con mayor calidad (tamaño del fruto uniforme, mayor grosor del pericarpio) que con la poda española. En todo caso, el aumento en los costos de mano de obra requerida para la implementación de la poda holandesa ha hecho que muchos productores no la adopten (Jovicich, Cantliffe y Stoffella, 2004). Se ha informado que, con la poda holandesa, se han obtenido rendimientos entre 100 y 200 $\mathrm{t} / \mathrm{ha}$, mientras que con la poda española se ha reportado entre 50 y 80 t/ha (Cruz-Huerta, Sánchez, Ortiz y Mendoza, 2009).

Es importante conocer la mejor densidad de siembra para cada variedad de chile dulce cultivado en invernadero, así como el mejor sistema de poda para esta, en cada sitio específico, con el fin de optimizar la producción y la rentabilidad. El objetivo de esta investigación fue evaluar el efecto de dos tipos de poda y tres densidades de siembra sobre el rendimiento y la calidad de un genotipo de chile dulce con frutos de forma cónica, cultivado bajo condiciones de invernadero en Alajuela, Costa Rica.

\section{Materiales y métodos}

La investigación se realizó en la Estación Experimental Agrícola Fabio Baudrit Moreno (EEAFBM) de la Universidad de Costa Rica, situada a $10^{\circ} 1^{\prime}$ latitud norte y $84^{\circ} 16^{\prime}$ longitud oeste, en el distrito San José del cantón Central de la provincia de Alajuela, a una altitud de $883 \mathrm{~m}$ s. n. m., con un promedio de precipitación anual de $1940 \mathrm{~mm}$ distribuidos de mayo a noviembre, y un promedio anual de temperatura de $22^{\circ} \mathrm{C}$.

El ensayo se llevó a cabo en un invernadero modelo XR de la marca Richel (Francia), tipo multicapilla, con techo de plástico, con ventilación cenital automática, y con un sistema de riego por goteo para proporcionar a las plantas el suministro de agua y nutrientes. El método de aplicación fue mediante goteros con una descarga de 2,0 1/hora por planta. Se utilizó un dosificador marca Dosatron con una proporción de inyección de 1:64. Se preparó la mezcla de sales solubles los días lunes, miércoles y viernes, a partir del día de trasplante. Los días viernes se preparó una mezcla aumentada en un $50 \%$ con respecto a los otros días, para nutrir al cultivo durante el fin de semana.

Se utilizó el genotipo de chile dulce (Capsicum annuum L.) cv. FBM-9, que corresponde a un híbrido F-1, con frutos de forma cónica, de color rojo a la madurez, producido por la Universidad de Costa Rica. La siembra del almácigo se realizó el 30 de junio de 2010, en bandejas plásticas de germinación de 128 alvéolos; se utilizó un sustrato compuesto por $50 \%$ de fibra de coco molida y $50 \%$ de abono orgánico marca Juan Viñas.

El trasplante se realizó el 7 de agosto del mismo año, 38 días después de la siembra. Las plántulas se establecieron en 168 sacos de $1 \mathrm{~m}$ de largo, $22 \mathrm{~cm}$ de ancho y $22 \mathrm{~cm}$ de altura, rellenos con sustrato inerte de fibra de coco molida. Previo al trasplante, los sacos se desinfectaron con el fungicida-bactericida Butrol (i.a. TCMTB), a una dosis de $2 \mathrm{ml} / \mathrm{l}$. Se evaluaron dos tipos de poda y tres densidades de siembra, para un total de seis tratamientos (Tabla 1).

La poda holandesa consiste en dirigir la planta a dos guías, eliminando posteriormente una de las dos bifurcaciones en que se va dividiendo cada rama (Gamayo, 1996), y la poda española consiste 
en dejar la planta a libre crecimiento (Jovicich, Cantliffe y Stoffella, 2004). La poda holandesa se aplicó inicialmente el 14 de setiembre de 2010, a los 38 días después del trasplante (ddt), y se siguió realizando periódicamente.

Las densidades de siembra de 2.60; 3.25; y 3.90 plantas $/ \mathrm{m}^{2}$ corresponden a cuatro, cinco y seis plantas por metro lineal (cada saco) respectivamente, es decir, una distancia entre plantas de 25.00; 20.00; y $16.67 \mathrm{~cm}$, respectivamente, y una distancia entre hileras de $1.54 \mathrm{~m}$.

La cosecha inició a los 79 ddt, y se realizó una o dos veces a la semana, hasta el 6 de abril de 2011 (242 ddt), recolectando los frutos que mostraban al menos un $50 \%$ de madurez. Los frutos cosechados se clasificaron de acuerdo con los parámetros de calidad que se muestran en la Tabla 2. Se registró el número de frutos de cada categoría de calidad, y se pesaron utilizando una balanza electrónica marca Ocony, modelo TH-IEK, de $5000.0 \pm 0.1 \mathrm{~g}$ de capacidad.

Se registraron los datos de temperatura y humedad relativa que prevalecieron dentro del invernadero, durante la ejecución del experimento, mediante sensores y un registrador de datos marca Onset, modelo HOBO U30.

\section{Tabla 1}

Tratamientos evaluados en el ensayo

\begin{tabular}{lcc}
\hline Tratamiento & Tipo de poda & Densidad de siembra $\left(\right.$ plantas $\left./ \mathrm{m}^{2}\right)$ \\
\hline 1 & Española & 2.60 \\
2 & Española & 3.25 \\
3 & Española & 3.90 \\
4 & Holandesa & 2.60 \\
5 & Holandesa & 3.25 \\
6 & Holandesa & 390 \\
\hline
\end{tabular}

Fuente. Elaboración propia.

Tabla 2

Parámetros de calidad utilizados en el ensayo

\begin{tabular}{lccc}
\hline & \multicolumn{3}{c}{ Categoría de calidad } \\
\cline { 2 - 4 } Parámetro & Primera & Segunda & Rechazo \\
\hline Longitud del fruto & Igual o mayor a $12 \mathrm{~cm}$ & Entre 6 y $12 \mathrm{~cm}$ & Menor a 6 cm \\
Forma del fruto & Cónica normal & Frutos “curveados" & Frutos deformes \\
$\begin{array}{l}\text { Presencia de manchas, cicatrices o } \\
\text { quema de sol }\end{array}$ & Ninguna & No mayores a $1 \mathrm{~cm}^{2}$ & Mayores a $1 \mathrm{~cm}^{2}$ \\
Estrías o grietas & Ninguna & No mayores a $3 \mathrm{~cm}$ & Mayores a 3 cm \\
Daño por plagas o enfermedades & Ninguno & Ninguno & Presente \\
\hline
\end{tabular}

Fuente. Elaboración propia. 


\section{Variables evaluadas}

Edad a inicio de cosecha. Se determinó registrando los días después del trasplante en que se cosechó el primer fruto con al menos un $50 \%$ de madurez.

Altura de la planta. Se midió la altura de las cuatro plantas de la unidad experimental al final del ciclo del cultivo (242 ddt), y se obtuvo el promedio. La altura se midió desde la base del tallo de la planta hasta el último meristemo apical, con la ayuda de una cinta métrica marca Assist, modelo 32G-8025, con una capacidad de $800.0 \mathrm{~cm}$ y una incertidumbre de $0.1 \mathrm{~cm}$.

Número de frutos por planta y por metro cuadrado. Se determinó el número de frutos por planta para cada categoría de calidad, realizando una sumatoria de los frutos obtenidos en todas las cosechas, y luego se dividió entre el número de plantas por parcela. Con base en este resultado y en la densidad de siembra, se calculó el número de frutos por metro cuadrado.

Peso promedio del fruto. Se determinó realizando una sumatoria del peso en gramos de todos los frutos registrados durante todas las cosechas, y luego se dividió entre el número de frutos totales; esta variable se determinó para cada categoría de calidad del fruto.

Rendimiento por hectárea. Se calculó a partir del peso de los frutos cosechados y de la densidad de siembra, para cada categoría de calidad del fruto. El rendimiento comercial se calculó sumando el peso de la producción de las categorías de primera y segunda calidad; el rendimiento total se obtuvo sumando el peso obtenido para las tres categorías de calidad.

\section{Diseño experimental}

El diseño experimental consistió en parcelas divididas; la parcela grande correspondió al tipo de poda, la parcela pequeña a la densidad de siembra y se utilizaron cuatro repeticiones.
La unidad experimental estuvo constituida por dos sacos con cuatro, cinco o seis plantas cada uno (según la densidad de siembra), y la parcela útil estuvo conformada por las cuatro plantas ubicadas en la posición central de esta. Para todas las variables, se realizó un análisis estadístico de variancia, y se utilizó la prueba LSD Fisher con una significancia de $5 \%$ para confirmar o descartar diferencias entre tratamientos.

\section{Resultados y discusión}

Durante el período en que se desarrolló el ensayo, la temperatura diurna promedio se ubicó en $27{ }^{\circ} \mathrm{C}$ (con extremos máximos de 37 ${ }^{\circ} \mathrm{C}$ ), y la temperatura nocturna promedio fue de $19{ }^{\circ} \mathrm{C}$ (con extremos mínimos de $14{ }^{\circ} \mathrm{C}$ ). La humedad relativa diurna promedio fue de $56 \%$ (con extremos mínimos de $20 \%$ ) y la humedad relativa nocturna promedio fue de $85 \%$ (con extremos máximos de 100\%). A partir de diciembre de 2010, aumentó la temperatura diurna y descendió la temperatura nocturna; también bajó la humedad relativa, tanto diurna como nocturna; estos cambios correspondieron al inicio y establecimiento de la temporada seca.

\section{Edad a inicio de cosecha}

La cosecha de chile dulce inició a los $79 \mathrm{ddt}$ para todos los tratamientos, y las plantas se mantuvieron en producción hasta los $242 \mathrm{ddt}$.

En San Carlos, Costa Rica, se informó que el inicio de la cosecha de chile dulce Nathalie, cultivado en invernadero, se produjo a los 58 ddt (Campos, 2009); esta mayor precocidad con respecto a los resultados obtenidos en la presente investigación es probable que se debiera a las mayores temperaturas que se registran normalmente en San Carlos, con respecto a las condiciones presente en la EEAFBM.

Por otra parte, en otros ensayos se han obtenido resultados similares a los hallados en el presente trabajo, con un inicio de la cosecha entre los 74 y 91 ddt (Aguado, Del Castillo, Uribarri, 
Galdeano y Sádaba, 2007; Montaño y Belisario, 2012; Elizondo-Cabalceta y Monge-Pérez, 2017).

Por otra parte, otros investigadores, al evaluar ocho cultivares de chile dulce tipo cuadrado y rectangular en Argentina, encontraron que el inicio de la cosecha se dio entre 124 y $128 \mathrm{ddt}$ (Iglesias, Roma y Pasini, s. f.). En México, al evaluar 13 híbridos de chile dulce tipo cuadrado en invernadero a $2240 \mathrm{~m} \mathrm{s.} \mathrm{n.} \mathrm{m.,} \mathrm{se} \mathrm{encontró}$ que el inicio de cosecha sucedió entre los $91 \mathrm{y}$ 111 ddt (Moreno, Mora, Sánchez y García-Pérez, 2011). En Colombia, se evaluaron seis genotipos de chile dulce con frutos de forma cónica, en un invernadero ubicado a $2650 \mathrm{~m}$ s. n. m., y encontraron queel inicio de la cosecha se presentó entre los 185 y los $213 \mathrm{ddt}$, según el genotipo, y para la variedad Nathalie esto sucedió a los 204 ddt (Monsalve, Casilimas y Bojacá, 2011). Dichos investigadores obtuvieron valores superiores a los resultados obtenidos en el presente trabajo para esta variable, lo cual puede deberse a las diferentes condiciones climáticas en que se realizaron las distintas evaluaciones, en especial la temperatura, que fue mayor en la EEAFBM.

\section{Altura de la planta}

No se presentaron diferencias significativas entre tratamientos para la variable altura de la planta a los $242 \mathrm{ddt}$; el valor promedio fue de $161.30 \mathrm{~cm}$.

En chile dulce, se tienen informes de que la altura de la planta puede variar entre 49,31 y 223,50 cm (Jovicich, Cantliffe y Hochmuth, 1999; Jovicich, Cantliffe y Stoffella, 2003; Zúñiga-Estrada y otros, 2004; Jovicich, Cantliffe y Stoffella, 2004; Grijalva-Contreras, MacíasDuarte y Robles-Contreras, 2008; Paunero, 2008; Campos, 2009; Reséndiz-Melgar, Moreno-Pérez, Sánchez-Del Castillo, Rodríguez-Pérez y PeñaLomelí, 2010; Moreno, Mora, Sánchez y GarcíaPérez, 2011; Quesada, 2015; Elizondo-Cabalceta y Monge-Pérez, 2016; Monge-Pérez, 2016); los resultados obtenidos en el presente trabajo se ubican en este rango.

En algunas ocasiones, la altura de la planta de chile dulce es mayor conforme aumenta la densidad de siembra (Jovicich, Cantliffe y Stoffella, 2004; Zúñiga-Estrada y otros, 2004; Seifi, Nemati, Shoor y Abedi, 2012), pero en otras, se ha encontrado el resultado contrario (Aminifard, Aroiee, Ameri y Fatemi, 2012), o no se han encontrado diferencias significativas entre diversas densidades de siembra (GrijalvaContreras, Macías-Duarte y Robles-Contreras, 2008; Reséndiz-Melgar, Moreno-Pérez, SánchezDel Castillo, Rodríguez-Pérez y Peña-Lomelí, 2010; Monge-Pérez, 2016), como sucedió en el presente ensayo.

Se ha informado que la altura de la planta de chile dulce es mayor en aquellas con poda holandesa (dos tallos por planta; $207 \mathrm{~cm}$ ) en comparación con plantas con poda española (que tienen más de dos tallos por planta; $190 \mathrm{~cm}$ ) (Jovicich, Cantliffe y Stoffella, 2004), mientras que en otros estudios no se encontraron diferencias significativas para esta variable entre la poda a dos y a cuatro tallos por planta (GrijalvaContreras, Macías-Duarte y Robles-Contreras, 2008), o entre la poda holandesa y la poda española (Monge-Pérez, 2016), lo que es similar al resultado obtenido en el presente ensayo.

Un investigador informó que la altura de la planta de chile dulce al final del ciclo del cultivo (177 ddt), al cultivar el híbrido Nathalie a una densidad de 1.67 plantas $/ \mathrm{m}^{2}$ en San Carlos, Costa Rica, varió entre 156.66 y 184.03 cm (Campos, 2009); los resultados obtenidos en el presente trabajo son similares a estos, aunque se utilizó otro genotipo.

En otro estudio, al evaluar el genotipo de chile dulce FBM-9 en la EEAFBM, a una densidad de 1.56 plantas $/ \mathrm{m}^{2}$, se encontró una altura de planta de $121.3 \mathrm{~cm}$ (Quesada, 2015); en la presente 
investigación, ese mismo genotipo obtuvo una mayor altura de planta $(161.3 \mathrm{~cm})$, lo cual se puede explicar debido a que se utilizaron mayores densidades de siembra.

\section{Número de frutos por planta}

En la Tabla 3, se presentan los datos del número de frutos por planta para los diferentes tratamientos. Se obtuvieron más frutos totales por planta con la poda española (29.71) que con la poda holandesa (19.21). Asimismo, se produjo una mayor cantidad de frutos totales por planta con las densidades de 2.60 plantas/ $\mathrm{m}^{2}$ y 3.25 plantas $/ \mathrm{m}^{2}$, en comparación con 3.90 plantas $/ \mathrm{m}^{2}$. Con respecto a los tratamientos, el mayor número de frutos totales por planta se obtuvo con la poda española, tanto a 2.60 plantas $/ \mathrm{m}^{2}$ como a 3.25 plantas $/ \mathrm{m}^{2}$ (31.94 y 31.69 frutos/planta, respectivamente); todos los demás tratamientos produjeron significativamente menos frutos por planta.

Según diferentes investigadores, el número total de frutos de chile dulce por planta puede variar entre 3.19 y 78.70 (Cebula, 1995; Jovicich, Cantliffe y Hochmuth, 1999; Quipildor, 2001; Aranguiz, 2002; Dasgan y Abak, 2003; Paunero, 2008; Campos, 2009; Cruz-Huerta, Sánchez, Ortiz y Mendoza, 2009; Reséndiz-Melgar, MorenoPérez, Sánchez-Del Castillo, Rodríguez-Pérez y

\section{Tabla 3}

Número de frutos por planta, según categoría de calidad, en chile dulce FBM-9

\begin{tabular}{|c|c|c|c|c|c|}
\hline \multirow[b]{2}{*}{ Efecto } & \multirow[b]{2}{*}{ Tratamiento } & \multicolumn{4}{|c|}{ Número de frutos por planta, según calidad } \\
\hline & & Total & Primera & Segunda & Rechazo \\
\hline \multirow[t]{2}{*}{ Tipo de poda } & Holandesa & $19.21 \mathrm{~b}$ & $8.56 \mathrm{~b}$ & $4.92 \mathrm{~b}$ & $5.81 \mathrm{~b}$ \\
\hline & Española & $29.71 \mathrm{a}$ & $9.85 \mathrm{a}$ & $12.96 \mathrm{a}$ & $6.96 \mathrm{a}$ \\
\hline \multirow{3}{*}{$\begin{array}{l}\text { Densidad } \\
\left(\text { plantas } / \mathrm{m}^{2}\right)\end{array}$} & 2.60 & $26.75 \mathrm{a}$ & $9.44 \mathrm{a}$ & $9.97 \mathrm{a}$ & $7.38 \mathrm{a}$ \\
\hline & 3.25 & $25.28 \mathrm{a}$ & $9.47 \mathrm{a}$ & $9.19 \mathrm{ab}$ & $6.69 \mathrm{a}$ \\
\hline & 3.90 & $21.34 \mathrm{~b}$ & $8.72 \mathrm{a}$ & $7.66 \mathrm{~b}$ & $5.09 \mathrm{~b}$ \\
\hline \multicolumn{2}{|c|}{$\begin{array}{l}\text { Interacción Tipo de poda } x \\
\text { Densidad }\end{array}$} & ns & ns & ns & ns \\
\hline \multicolumn{6}{|c|}{ Tipo de poda $x$ Densidad (plantas $/ \mathrm{m}^{2}$ ) } \\
\hline \multirow[t]{3}{*}{ Española } & 2.60 & $31.94 \mathrm{a}$ & ns & $14.75 \mathrm{a}$ & $7.56 \mathrm{a}$ \\
\hline & 3.25 & $31.69 \mathrm{a}$ & ns & $13.75 \mathrm{a}$ & $7.50 \mathrm{a}$ \\
\hline & 3.90 & $25.69 \mathrm{~b}$ & ns & $10.38 \mathrm{~b}$ & $5.81 \mathrm{ab}$ \\
\hline \multirow[t]{3}{*}{ Holandesa } & 2.60 & $21.63 \mathrm{bc}$ & ns & $5.19 \mathrm{c}$ & $7.19 \mathrm{a}$ \\
\hline & 3.25 & $19.01 \mathrm{c}$ & ns & $4.63 \mathrm{c}$ & $5.88 \mathrm{ab}$ \\
\hline & 3.90 & $17.26 \mathrm{c}$ & ns & $4.94 \mathrm{C}$ & $4.38 \mathrm{~b}$ \\
\hline
\end{tabular}

Nota. Datos que comparten una misma letra no presentan diferencias significativas ( $p \leq .05)$, según la prueba LSD Fisher (ns: no significativa. Fuente: Elaboración propia, a partir de los datos obtenidos. 
Peña-Lomelí, 2010; Sharma, Semwal y Uniyal, 2010; Moreno, Mora, Sánchez y García-Pérez, 2011; Borosic, y otros, 2012; Montaño y Belisario, 2012; Wahb-Allah, 2013; Quesada, 2015; MongePérez, 2016; Elizondo-Cabalceta y Monge-Pérez, 2017); los resultados obtenidos en la presente investigación se ubican dentro de este rango.

En chile dulce, generalmente esta característica muestra valores mayores conforme disminuye la densidad de siembra (Cebula, 1995; Jovicich, Cantliffe y Hochmuth, 1999; Dasgan y Abak, 2003; Cruz-Huerta, Sánchez, Ortiz y Mendoza, 2009; Reséndiz-Melgar, Moreno-Pérez, SánchezDel Castillo, Rodríguez-Pérez y Peña-Lomelí, 2010; Monge-Pérez, 2016), y conforme se dejan más tallos por planta como en el caso de la poda española (Cebula, 1995; Jovicich, Cantliffe y Hochmuth, 1999; Dasgan y Abak, 2003; Seifi, Nemati, Shoor y Abedi, 2012; Monge-Pérez, 2016), tal y como se evidenció también en el presente ensayo. Sin embargo, otro investigador no encontró diferencias significativas entre tratamientos para esta variable al evaluar dos, tres y cuatro tallos por planta (Aranguiz, 2002).

En un ensayo, se obtuvo entre 28.7 y 39.2 frutos totales por planta en el cultivo de chile dulce en invernadero, a una densidad de 1.67 plantas/ $\mathrm{m}^{2}$ (Campos, 2009); esos valores son superiores a los obtenidos en el presente ensayo, debido probablemente, entre otros factores, a que se utilizaron densidades de siembra mayores en la presente investigación.

Varios investigadores han obtenido menos de 10 frutos totales por planta en chile dulce. En un ensayo, al evaluar el efecto de la densidad de siembra y el número de tallos por planta en un chile dulce tipo cuadrado en invernadero, se encontró que el mayor número de frutos por planta (6.47) se alcanzó con 2 plantas $/ \mathrm{m}^{2}$ (en vez de 3 o 4 plantas $/ \mathrm{m}^{2}$ ) y en plantas con cuatro tallos (en vez de uno o dos) (Jovicich, Cantliffe y Hochmuth, 1999). En otro estudio, al evaluar 17 variedades de chile dulce tipo cuadrado a una densidad de 4.0 plantas $/ \mathrm{m}^{2}$, se encontró que el número total de frutos por planta varió entre 4.75 y 7.63 (Reséndiz-Melgar, Moreno-Pérez, Sánchez-Del Castillo, Rodríguez-Pérez y PeñaLomelí, 2010). Otros investigadores, al evaluar 23 genotipos de chile dulce tipo rectangular a campo abierto, encontraron una producción total entre 3.83 y 9.30 frutos/planta (Sharma, Semwal y Uniyal, 2010). Todos estos datos son muy inferiores a los conseguidos en el presente ensayo, pero se debe tomar en cuenta que generalmente las variedades de chile dulce con frutos de forma cuadrada o rectangular producen menos frutos por planta que las variedades con frutos de forma cónica (J. E. Monge-Pérez, datos sin publicar).

En cuanto a la producción de frutos de primera calidad (Tabla 1), se obtuvo un número significativamente superior de frutos por planta con poda española que con poda holandesa; sin embargo, no se presentaron diferencias significativas entre tratamientos para esta variable. Con respecto a los frutos de segunda calidad, se obtuvo mayor cantidad de frutos por planta con la poda española en comparación con la poda holandesa, y con la densidad de 2.60 plantas $/ \mathrm{m}^{2}$ en relación con 3.90 plantas $/ \mathrm{m}^{2}$.

En Chile, al cultivar dos genotipos de chile dulce a 3.1 plantas $/ \mathrm{m}^{2}$, no se encontró diferencias entre la poda de la planta a dos, tres o cuatro tallos, para la variable número de frutos totales por planta (entre 20.9 y 21.7), ni para el número de frutos comerciales por planta (entre 15.6 y 18.2) (Aranguiz, 2002).

En relación con la producción de frutos comerciales, en un estudio en Croacia, al evaluar cinco genotipos de chile dulce, se encontró una producción entre 34 y 53 frutos comerciales por planta (Borosic, y otros, 2012). Por otra parte, otro investigador, al evaluar 22 genotipos de chile dulce tipo rectangular en invernadero en 
Argentina, a una densidad de 2.1 plantas $/ \mathrm{m}^{2}$, encontró que el número de frutos comerciales por planta osciló entre 37 y 50, durante un ciclo de cosecha de 189 días (Quipildor, 2001); estos datos son superiores a los encontrados en la presente investigación (entre 13.48 y 22.81 frutos comerciales/planta), aunque es necesario tomar en cuenta las diferencias en densidad de siembra, criterios de calidad, clima y genotipo entre los diferentes ensayos.

En otro estudio en Argentina, se evaluaron nueve genotipos de chile dulce tipo rectangular y cuadrado, cultivados a una densidad de 1.56 plantas $/ \mathrm{m}^{2}$, y se halló una producción total de entre 13.21 y 34.1 frutos/planta, que incluyó entre 9.01 y 23.13 frutos de primera calidad y entre 3.01 y 7.48 frutos de segunda calidad por planta (Paunero, 2008); estos datos se acercan a los obtenidos en la presente investigación, aunque los genotipos, las densidades de siembra y los criterios de calidad utilizados fueron diferentes.

El número de frutos producidos por planta depende también de su tamaño. Por ejemplo, en un estudio se evaluaron 36 genotipos de chile dulce en invernadero, a una densidad de 2.78 plantas $/ \mathrm{m}^{2}$, y se encontró que el número de frutos comerciales por planta varió entre 10.8 para los genotipos de frutos grandes (diámetro mayor a $64 \mathrm{~mm}$ ) y 20.0 para los genotipos de frutos pequeños (diámetro menor a $64 \mathrm{~mm}$ ), aunque el rendimiento comercial fue similar para ambos grupos (entre 2.6 y $3.4 \mathrm{~kg} /$ planta) (Shaw y Cantliffe, 2002).

En otro ensayo, al evaluar 27 genotipos de chile dulce tipo cuadrado cultivado a campo abierto, se obtuvo entre 0.19 y 8.02 frutos comerciales por planta (Hutton y Handley, 2007). Estos datos son inferiores a los obtenidos en este trabajo, debido a que la producción de frutos por planta generalmente es más alta en invernadero que a campo abierto.
Otro investigador, al evaluar el genotipo de chile dulce FBM-9 en la EEAFBM, a una densidad de 1.56 plantas $/ \mathrm{m}^{2}$, y al utilizar las mismas categorías de calidad empleadas en el presente trabajo, obtuvo 29.4 frutos totales por planta, que incluyeron 5.3 frutos de primera calidad y 12.0 frutos de segunda calidad por planta (Quesada, 2015). En contraste, en la presente investigación se obtuvo una mayor cantidad de frutos de primera calidad por planta, a pesar de utilizar densidades de siembra mayores.

\section{Peso promedio del fruto}

En la Tabla 4 se presentan los datos de peso promedio del fruto, según su calidad. La densidad de siembra no afectó el peso promedio del fruto en ninguna de las categorías de calidad. Con respecto al tipo de poda, se mostró un mayor peso promedio total del fruto con la poda holandesa (95.15 g) que con la poda española (85.69 g); lo mismo sucedió para todas las categorías de calidad del fruto. En relación con los tratamientos, se encontró un mayor peso promedio total en los frutos de las plantas a las cuales se les practicó poda holandesa en las densidades de 3.25 y 3.90 plantas $/ \mathrm{m}^{2}$, con respecto a las plantas que recibieron poda española en cualquiera de las densidades de siembra evaluadas.

En chile dulce se tienen informes de que el peso promedio del fruto puede variar entre 25.5 y 323.0 g (Cebula, 1995; Lorenzo y Castilla, 1995; Quipildor, 2001; Aranguiz, 2002; Shaw y Cantliffe, 2002; Dasgan y Abak, 2003; Rotondo, Mondino, Ferratto, Grasso y Longo, 2003; Vicente-Conesa, Condés-Rodríguez, Sáez-García y García-García, 2005; GrijalvaContreras, Macías-Duarte, Robles-Contreras y Valenzuela-Ruiz, 2006; Aguado, Del Castillo, Uribarri, Galdeano y Sádaba, 2007; Hutton y Handley, 2007; Grijalva-Contreras, MacíasDuarte y Robles-Contreras, 2008; Paunero, 2008; Campos, 2009; Cruz-Huerta, Sánchez, Ortiz 
Tabla 4

Peso promedio del fruto, según categoría de calidad, en chile dulce FBM-9

\begin{tabular}{|c|c|c|c|c|c|}
\hline \multirow{2}{*}{ Efecto } & \multirow[b]{2}{*}{ Tratamiento } & \multicolumn{4}{|c|}{ Peso promedio del fruto (gramos), según calidad } \\
\hline & & Total & Primera & Segunda & Rechazo \\
\hline \multirow[t]{2}{*}{ Tipo de poda } & Holandesa & $95.15 \mathrm{a}$ & $115.09 \mathrm{a}$ & $86.46 \mathrm{a}$ & $71.93 \mathrm{a}$ \\
\hline & Española & $85.69 \mathrm{~b}$ & $108.76 \mathrm{~b}$ & $77.69 \mathrm{~b}$ & $65.65 \mathrm{~b}$ \\
\hline \multirow{3}{*}{$\begin{array}{l}\text { Densidad } \\
\left(\text { plantas } / \mathrm{m}^{2}\right)\end{array}$} & 2.60 & $88.15 \mathrm{a}$ & $109.80 \mathrm{a}$ & $84.14 \mathrm{a}$ & $66.50 \mathrm{a}$ \\
\hline & 3.25 & 90.49 a & $111.23 \mathrm{a}$ & $80.49 \mathrm{a}$ & $71.65 \mathrm{a}$ \\
\hline & 3.90 & $92.63 \mathrm{a}$ & $114.75 \mathrm{a}$ & $81.60 \mathrm{a}$ & $68.23 \mathrm{a}$ \\
\hline \multirow{2}{*}{\multicolumn{2}{|c|}{$\begin{array}{l}\text { Interacción Tipo de poda x } \\
\text { Densidad }\end{array}$}} & ns & ns & ns & ns \\
\hline & & de poda $x$ & ensidad $(p$ & $\left./ \mathrm{m}^{2}\right)$ & \\
\hline \multirow[t]{3}{*}{ Española } & 2.60 & $82.93 \mathrm{~d}$ & $105,43 \mathrm{~b}$ & $77.48 \mathrm{bc}$ & $63.33 \mathrm{~b}$ \\
\hline & 3.25 & $84.70 \mathrm{~cd}$ & $107,58 \mathrm{ab}$ & $77.13 \mathrm{c}$ & $66.45 \mathrm{~b}$ \\
\hline & 3.90 & 89.45 bc & $113,28 \mathrm{ab}$ & 78.48 bc & $67.18 \mathrm{ab}$ \\
\hline \multirow[t]{3}{*}{ Holandesa } & 2.60 & $93.38 \mathrm{ab}$ & $114.18 \mathrm{ab}$ & $90.80 \mathrm{a}$ & $69.68 \mathrm{ab}$ \\
\hline & 3.25 & $96.28 \mathrm{a}$ & $114.88 \mathrm{a}$ & $83.85 \mathrm{abc}$ & 76.85 a \\
\hline & 3.90 & $95.80 \mathrm{a}$ & $116.23 \mathrm{a}$ & $84,73 \mathrm{ab}$ & $69.28 \mathrm{ab}$ \\
\hline
\end{tabular}

Nota. Datos que comparten una misma letra no presentan diferencias significativas ( $p \leq .05)$, según la prueba LSD Fisher (ns: no significativa. Fuente: Elaboración propia, a partir de los datos obtenidos.

y Mendoza, 2009; Macua, Lahoz, Calvillo y Orcaray, 2010; Reséndiz-Melgar, Moreno-Pérez, Sánchez-Del Castillo, Rodríguez-Pérez y PeñaLomelí, 2010; Sharma, Semwal y Uniyal, 2010; Moreno, Mora, Sánchez y García-Pérez, 2011; Borosic y otros, 2012; Montaño y Belisario, 2012; Seifi, Nemati, Shoor y Abedi, 2012; Wahb-Allah, 2013; Mahmoud y El-Eslamboly, 2015; MongePérez, 2016; Elizondo-Cabalceta y Monge-Pérez, 2017); los resultados obtenidos en la presente investigación se ubican dentro de dicho rango.

En algunas investigaciones, esta variable ha mostrado valores mayores conforme disminuye la densidad de siembra del chile dulce (CruzHuerta, Sánchez, Ortiz y Mendoza, 2009; Aminifard, Aroiee, Ameri y Fatemi, 2012; Seifi, Nemati, Shoor y Abedi, 2012), aunque en otras no se presentaron diferencias entre distintas densidades de siembra (Dasgan y Abak, 2003; Grijalva-Contreras, Macías-Duarte y RoblesContreras, 2008; Reséndiz-Melgar, MorenoPérez, Sánchez-Del Castillo, Rodríguez-Pérez y Peña-Lomelí, 2010; Monge-Pérez, 2016), tal y como sucedió en la presente investigación.

Por otra parte, otros investigadores mencionan que el peso promedio del fruto de chile dulce es 
mayor conforme disminuye el número de tallos por planta (Rotondo, Mondino, Ferratto, Grasso y Longo, 2003; Seifi, Nemati, Shoor y Abedi, 2012; Monge-Pérez, 2016), lo que constituye una mejora en su calidad, tal y como sucedió en el presente ensayo, el cual fue mayor con poda holandesa en comparación con poda española. Sin embargo, otros autores no han encontrado diferencias significativas para esta variable al evaluar diferente número de tallos por planta (Aranguiz, 2002; Dasgan y Abak, 2003).

En otro estudio, al evaluar el genotipo de chile dulce FBM-9 en la EEAFBM, a una densidad de 1.56 plantas $/ \mathrm{m}^{2}$, y al utilizar los mismos parámetros de calidad empleados en la presente investigación, se encontró que el peso promedio del fruto fue de $114.8 \mathrm{~g}$ en la calidad de primera, $79.8 \mathrm{~g}$ en la calidad de segunda, y 50.3 en la calidad de rechazo (Quesada, 2015); estos resultados son muy similares a los obtenidos en el presente trabajo.

\section{Rendimiento por hectárea}

Se obtuvo un mayor rendimiento total y comercial con la poda española (81.60 y $66.98 \mathrm{t}$ / ha, respectivamente) que con la poda holandesa (58.48 y $45.24 \mathrm{t} / \mathrm{ha}$, respectivamente) (Tabla 5). Asimismo, el rendimiento total y comercial fue más alto con las densidades de 3.25 y 3.90 plantas/

\section{Tabla 5}

Rendimiento por hectárea, según categoría de calidad, en chile dulce FBM-9

\begin{tabular}{|c|c|c|c|c|c|c|}
\hline \multirow[b]{2}{*}{ Efecto } & \multirow[b]{2}{*}{ Tratamiento } & \multicolumn{5}{|c|}{ Rendimiento hasta los $242 \mathrm{ddt}(\mathrm{t} / \mathrm{ha})$, según calidad } \\
\hline & & Total & Comercial & Primera & Segunda & Rechazo \\
\hline \multirow[t]{2}{*}{ Tipo de poda } & Holandesa & $58.48 \mathrm{~b}$ & $45.24 \mathrm{~b}$ & $31.56 \mathrm{a}$ & $13.68 \mathrm{~b}$ & $13.25 \mathrm{a}$ \\
\hline & Española & $81.60 \mathrm{a}$ & $66.98 \mathrm{a}$ & $34.98 \mathrm{a}$ & $32.00 \mathrm{a}$ & $14.63 \mathrm{a}$ \\
\hline \multirow{3}{*}{$\begin{array}{l}\text { Densidad } \\
\left(\text { plantas } / \mathrm{m}^{2}\right)\end{array}$} & 2.60 & $60.51 \mathrm{~b}$ & $47.78 \mathrm{~b}$ & $26.72 \mathrm{~b}$ & $21.06 \mathrm{a}$ & $12.74 \mathrm{a}$ \\
\hline & 3.25 & $73.06 \mathrm{a}$ & $57.70 \mathrm{a}$ & $34.18 \mathrm{a}$ & $23.52 \mathrm{a}$ & $15.37 \mathrm{a}$ \\
\hline & 3.90 & $76.54 \mathrm{a}$ & $62.84 \mathrm{a}$ & 38.91 a & $23.93 \mathrm{a}$ & $13.71 \mathrm{a}$ \\
\hline \multicolumn{2}{|c|}{$\begin{array}{l}\text { Interacción Tipo de poda } x \\
\text { Densidad }\end{array}$} & ns & ns & ns & ns & \\
\hline \multicolumn{7}{|c|}{ Tipo de poda $\times$ Densidad (plantas $/ \mathrm{m}^{2}$ ) } \\
\hline \multirow[t]{3}{*}{ Española } & 2.60 & $68.50 \mathrm{~b}$ & $56,10 \mathrm{~b}$ & $26,30 \mathrm{~d}$ & ns & ns \\
\hline & 3.25 & $87.10 \mathrm{a}$ & $71,00 \mathrm{a}$ & $36,60 \mathrm{ab}$ & ns & ns \\
\hline & 3.90 & $89.20 \mathrm{a}$ & 73,80 a & $42,00 \mathrm{a}$ & ns & ns \\
\hline \multirow[t]{3}{*}{ Holandesa } & 2.60 & $52.40 \mathrm{c}$ & $39.40 \mathrm{c}$ & $27.10 \mathrm{~cd}$ & ns & ns \\
\hline & 3.25 & $59.10 \mathrm{bc}$ & $44.40 \mathrm{bc}$ & $31.70 \mathrm{bcd}$ & ns & ns \\
\hline & 3.90 & $64.00 \mathrm{bc}$ & $51.90 \mathrm{~b}$ & $35.80 \mathrm{abc}$ & ns & ns \\
\hline
\end{tabular}

Nota. Datos que comparten una misma letra no presentan diferencias significativas ( $p \leq .05)$, según la prueba LSD Fisher (ns: no significativa. Fuente: Elaboración propia, a partir de los datos obtenidos. 
$\mathrm{m}^{2}$ que con 2.60 plantas $/ \mathrm{m}^{2}$. También se obtuvo un mayor rendimiento de frutos de primera calidad con las densidades de 3.25 y 3.90 plantas/ $\mathrm{m}^{2}$ que con 2.60 plantas $/ \mathrm{m}^{2}$. En cuanto a los tratamientos, la producción total y comercial fue significativamente más alta para las densidades de 3.25 y 3.90 plantas $/ \mathrm{m}^{2}$ con poda española, en comparación con los demás tratamientos.

Según diversas investigaciones, el rendimiento total en chile dulce puede variar entre $7.67 \mathrm{y}$ 178.4 t/ha (Iglesias, Roma y Pasini, s. f.; Cebula, 1995; Lorenzo y Castilla, 1995; Jovicich, Cantliffe y Hochmuth, 1999; Quipildor, 2001; Shaw y Cantliffe, 2002; Dasgan y Abak, 2003; Jovicich, Cantliffe y Stoffella, 2003; Rotondo, Mondino, Ferratto, Grasso y Longo, 2003; Jovicich, Cantliffe y Stoffella, 2004; Vicente-Conesa y Sáez-García, 2004; Vicente-Conesa, Condés-Rodríguez, Sáez-García y García-García, 2005; GrijalvaContreras, Macías-Duarte, Robles-Contreras y Valenzuela-Ruiz, 2006; Aguado, Del Castillo, Uribarri, Galdeano y Sádaba, 2007; GrijalvaContreras, Macías-Duarte y Robles-Contreras, 2008; Paunero, 2008; Cruz-Huerta, Sánchez, Ortiz y Mendoza, 2009; Macua, Lahoz, Calvillo y Orcaray, 2010; Maniutiu, Sima, Apahidean, Apahidean y Ficior, 2010; Reséndiz-Melgar, Moreno-Pérez, Sánchez-Del Castillo, RodríguezPérez y Peña-Lomelí, 2010; Monsalve, Casilimas y Bojacá, 2011; Moreno, Mora, Sánchez y García-Pérez, 2011; Aminifard, Aroiee, Ameri y Fatemi, 2012; Borosic y otros, 2012; Maboko, Du Plooy y Chiloane, 2012; Seifi, Nemati, Shoor y Abedi, 2012; Wahb-Allah, 2013; Mahmoud y ElEslamboly, 2015; Monge-Pérez, 2016; ElizondoCabalceta y Monge-Pérez, 2017); los resultados obtenidos en la presente investigación se ubican dentro de dicho rango. Sin embargo, otros investigadores, al evaluar un genotipo de chile dulce a una densidad de entre 12.0 y 24.0 plantas/ $\mathrm{m}^{2}$, obtuvieron rendimientos mucho más altos que los conseguidos en el presente ensayo (entre 342.6 y 398.4 t/ha) (Zúñiga-Estrada, y otros, 2004).
Generalmente, el rendimiento total en chile dulce es mayor conforme aumenta la densidad de siembra (Cebula, 1995; Lorenzo y Castilla, 1995; Jovicich, Cantliffe y Hochmuth, 1999; Dasgan y Abak, 2003; Rotondo, Mondino, Ferratto, Grasso y Longo, 2003; Jovicich, Cantliffe y Stoffella, 2004; Zúñiga-Estrada y otros, 2004; Maniutiu, Sima, Apahidean, Apahidean y Ficior, 2010; Aminifard, Aroiee, Ameri y Fatemi, 2012; Maboko, Du Plooy y Chiloane, 2012; Seifi, Nemati, Shoor y Abedi, 2012; Monge-Pérez, 2016), tal y como sucedió en la presente investigación. Sin embargo, en otros ensayos no se han determinado diferencias significativas para esta variable entre distintas densidades de siembra (Vicente-Conesa y SáezGarcía, 2004; Grijalva-Contreras, Macías-Duarte y Robles-Contreras, 2008; Reséndiz-Melgar, Moreno-Pérez, Sánchez-Del Castillo, RodríguezPérez y Peña-Lomelí, 2010).

Por otra parte, en varias investigaciones se ha encontrado que el rendimiento total en chile dulce es mayor conforme aumenta el número de tallos por planta (Jovicich, Cantliffe y Hochmuth, 1999; Dasgan y Abak, 2003; Rotondo, Mondino, Ferratto, Grasso y Longo, 2003; Vicente-Conesa, Condés-Rodríguez, Sáez-García y García-García, 2005; Maboko, Du Plooy y Chiloane, 2012; Seifi, Nemati, Shoor y Abedi, 2012; Monge-Pérez, 2016), tal y como sucedió en el presente ensayo, en que el rendimiento fue mayor con poda española que con poda holandesa, lo cual se asocia a una mayor eficiencia de la planta debido a un mayor índice de área foliar (Dasgan y Abak, 2003); sin embargo, en otras ocasiones se han encontrado resultados contrarios (Cebula, 1995; Rotondo, Mondino, Ferratto, Grasso y Longo, 2003), o no se han hallado diferencias al utilizar distinto número de tallos por planta (VicenteConesa y Sáez-García, 2004; Grijalva-Contreras, Macías-Duarte y Robles-Contreras, 2008).

En un estudio, al evaluar el chile dulce Nathalie en invernadero en San Carlos, Costa 
Rica, a 1.67 plantas $/ \mathrm{m}^{2}$, se obtuvo un rendimiento total entre 53.34 y 71.01 t/ha (Campos, 2009); en la presente investigación se obtuvieron valores mayores a esos en los tratamientos de poda española a 3.25 y 3.90 plantas $/ \mathrm{m}^{2}(87.10$ y $89.20 \mathrm{t} / \mathrm{ha}$, respectivamente), lo cual podría explicarse en parte por las mayores densidades de siembra utilizadas en el presente ensayo.

En España, se evaluaron ocho genotipos de chile dulce alargado (tipo Lamuyo) y se encontró un rendimiento total entre $144.2 \mathrm{y}$ $178.4 \mathrm{t} / \mathrm{ha}$, y un rendimiento de primera calidad entre 107.6 y 157.0 t/ha (Aguado, Del Castillo, Uribarri, Galdeano y Sádaba, 2007); en ese caso, la producción se extendió hasta los 281 ddt (200 días en cosecha). Esos resultados son superiores al rendimiento máximo obtenido en el presente ensayo (89.2 $t / h a)$, aunque se toma en cuenta que el ciclo del cultivo fue de solo 242 ddt (163 días en cosecha).

En Irán, varios investigadores encontraron que el rendimiento total de chile dulce cultivado al aire libre fue mayor a una densidad de 10,00 plantas/ $\mathrm{m}^{2}$ (164.1 t/ha), y se redujo, conforme disminuyó la densidad de siembra, hasta un mínimo de 49.1 $\mathrm{t} / \mathrm{ha}$ a una densidad de 3.33 plantas $/ \mathrm{m}^{2}$; por otra parte, el rendimiento por planta fue mayor con la densidad de 3.33 plantas $/ \mathrm{m}^{2}$ (1842 g/planta), y fue menor a una densidad de 6.67 plantas/ $\mathrm{m}^{2}$ (1285 g/planta) (Aminifard, Aroiee, Ameri y Fatemi, 2012); esto concuerda con lo hallado en otro estudio, en el que se indica que el aumento en la densidad de población causó disminución en el rendimiento por planta, pero incrementó el rendimiento por unidad de superficie (CruzHuerta, Sánchez, Ortiz y Mendoza, 2009).

En EE. UU., varios autores hallaron que el rendimiento total en chile dulce aumentó conforme se incrementó la densidad de siembra (Jovicich, Cantliffe y Hochmuth, 1999). También encontraron que las plantas con 4 tallos tuvieron un mayor rendimiento total (18.45 $\mathrm{t} / \mathrm{ha})$ que las plantas con solo 1 o 2 tallos (7.67 y 13.63 t/ha, respectivamente). El mayor rendimiento total (16.93 t/ha) se obtuvo con 2 plantas $/ \mathrm{m}^{2}$ (en vez de 3 o 4 plantas $/ \mathrm{m}^{2}$ ) y con plantas con 4 tallos (en vez de 1 o 2); el rendimiento total obtenido en la presente investigación fue mucho más alto que el obtenido por dichos investigadores.

En Colombia, se evaluaron genotipos de chile dulce tipo cuadrado y tipo cónico, a una densidad de 2.3 plantas $/ \mathrm{m}^{2}$, y se encontró que los tipo cuadrado tuvieron una productividad total entre 68 y 79 t/ha, mientras que los tipo cónico produjeron entre 31 y $48 \mathrm{t} / \mathrm{ha}$, y el genotipo Nathalie produjo $43 \mathrm{t} / \mathrm{ha}$ (Monsalve, Casilimas y Bojacá, 2011); en la presente investigación, el rendimiento total obtenido para todos los tratamientos (entre 52.40 y $89.20 \mathrm{t} / \mathrm{ha}$ ) fue superior al obtenido en dicho estudio por los genotipos de tipo cónico. Además, curiosamente dichos investigadores encontraron que los genotipos con frutos de color rojo produjeron significativamente menos (entre 31 y $48 \mathrm{~T} / \mathrm{ha}$ ) que aquellos con frutos de otros colores (entre 68 y 79 t/ha).

A campo abierto, los rendimientos de chile dulce generalmente son menores que en invernadero, pues se ha informado de valores de producción total entre 6.07 y 25.64 t/ha (Sharma, Semwal y Uniyal, 2010; Montaño y Belisario, 2012). Esos resultados son mucho más bajos que los obtenidos en la presente investigación.

Con respecto al rendimiento comercial en chile dulce, diversos investigadores han informado que este varía entre 10.28 y $74.00 \mathrm{t} / \mathrm{ha}$ (Iglesias, Roma y Pasini, s. f.; Lorenzo y Castilla, 1995; Jovicich, Cantliffe y Stoffella, 2004; Paunero, 2008; Cruz-Huerta, Sánchez, Ortiz y Mendoza, 2009; Macua, Lahoz, Calvillo y Orcaray, 2010; Maboko, Du Plooy y Chiloane, 2012; WahbAllah, 2013); los resultados obtenidos en el presente ensayo (entre 39.40 y $73.80 \mathrm{t} / \mathrm{ha}$ ) se ubican dentro de ese rango. 
En chile dulce, generalmente el rendimiento comercial es mayor conforme aumenta la densidad de siembra (Lorenzo y Castilla, 1995; Jovicich, Cantliffe y Stoffella, 2004; Cruz-Huerta, Sánchez, Ortiz y Mendoza, 2009; Maboko, Du Plooy y Chiloane, 2012); esto mismo se presentó en la presente investigación.

Por otra parte, el rendimiento comercial de chile dulce obtenido en la presente investigación también coincide con lo informado por otros autores, quienes encontraron mayores valores para esta variable al aumentar el número de tallos por planta (en este caso, con la poda española) (Maboko, Du Plooy y Chiloane, 2012). Por el contrario, en otro estudio no se encontraron diferencias significativas en el rendimiento comercial entre plantas tratadas con poda española y plantas tratadas con poda holandesa (Jovicich, Cantliffe y Stoffella, 2004).

En otra investigación, al evaluar 27 genotipos de chile dulce tipo cuadrado cultivado a campo abierto a 4.78 plantas $/ \mathrm{m}^{2}$, se obtuvo un rendimiento comercial entre 1.91 y $35.85 \mathrm{t} / \mathrm{ha}$ (Hutton y Handley, 2007); estos resultados son inferiores a los obtenidos en el presente ensayo, probablemente por tratarse de producción a campo abierto y no en invernadero.

Otro autor, al evaluar el genotipo de chile dulce FBM-9 en la EEAFBM, a una densidad de 1,56 plantas $/ \mathrm{m} 2$, encontró que el rendimiento comercial fue de 28,0 t/ha (Quesada, 2015). Esos resultados son mucho más bajos que los obtenidos en el presente estudio para ese mismo genotipo (entre 39,40 y 73,80t/ha), probablemente debido a que en el presente ensayo se utilizaron mayores densidades de siembra.

\section{Número de frutos por metro cuadrado}

En la Tabla 6, se presentan los datos del número de frutos por metro cuadrado para los diferentes tratamientos. Se obtuvo una mayor producción total con la poda española (95.19 frutos $\left./ \mathrm{m}^{2}\right)$ que con la poda holandesa (61.45 frutos $/ \mathrm{m}^{2}$ ); lo mismo sucedió con la producción de frutos de primera y de segunda calidad. También el resultado fue un mayor número de frutos totales y de primera calidad por metro cuadrado con las densidades de 3.25 y 3.90 plantas $/ \mathrm{m}^{2}$, en comparación con 2.60 plantas/ $\mathrm{m}^{2}$. Los tratamientos con mayor producción total fueron poda española a las densidades de 3.25 y 3.90 plantas $/ \mathrm{m}^{2}$ (102.99 y 99.70 frutos $/ \mathrm{m}^{2}$, respectivamente). El número de frutos por metro cuadrado es la variable más importante a nivel económico, dado que en Costa Rica el chile dulce se vende por unidad (cada fruto) y no por peso; además, los frutos de primera calidad se venden a un mayor precio que los de categoría de calidad inferior (J. E. Monge-Pérez, datos sin publicar).

En chile dulce se tienen informes de que el número total de frutos por metro cuadrado puede variar entre 7.0 y 320.24 (Cebula, 1995; Jovicich, Cantliffe y Hochmuth, 1999; Aranguiz, 2002; Shaw y Cantliffe, 2002; Dasgan y Abak, 2003; Jovicich, Cantliffe y Stoffella, 2003; Jovicich, Cantliffe y Stoffella, 2004; Cruz-Huerta, Sánchez, Ortiz y Mendoza, 2009; Reséndiz-Melgar, Moreno-Pérez, Sánchez-Del Castillo, RodríguezPérez y Peña-Lomelí, 2010; Monge-Pérez, 2016); los resultados obtenidos en la presente investigación se ubican dentro de este rango.

Generalmente, el número total de frutos por metro cuadrado en chile dulce es mayor conforme aumenta la densidad de siembra (Cebula, 1995; Jovicich, Cantliffe y Hochmuth, 1999; Dasgan y Abak, 2003; Jovicich, Cantliffe y Stoffella, 2004; Cruz-Huerta, Sánchez, Ortiz y Mendoza, 2009; Monge-Pérez, 2016), tal y como sucedió también en el presente estudio; sin embargo, otros autores no encontraron diferencias entre diversas densidades de siembra para esta variable (Reséndiz-Melgar, MorenoPérez, Sánchez-Del Castillo, Rodríguez-Pérez y Peña-Lomelí, 2010). 
Tabla 6

Número de frutos por metro cuadrado, según categoría de calidad, en chile dulce FBM-9.

\begin{tabular}{|c|c|c|c|c|c|}
\hline \multirow[b]{2}{*}{ Efecto } & \multirow[b]{2}{*}{ Tratamiento } & \multicolumn{4}{|c|}{ Número de frutos $/ \mathrm{m}^{2}$, según calidad } \\
\hline & & Total & Primera & Segunda & Rechazo \\
\hline \multirow[t]{2}{*}{ Tipo de poda } & Holandesa & $61.45 \mathrm{~b}$ & $27.55 \mathrm{~b}$ & $15.93 \mathrm{~b}$ & $18.28 \mathrm{a}$ \\
\hline & Española & $95.19 \mathrm{a}$ & $32.00 \mathrm{a}$ & $41.17 \mathrm{a}$ & $22.24 \mathrm{a}$ \\
\hline \multirow{3}{*}{$\begin{array}{l}\text { Densidad } \\
\left(\text { plantas } / \mathrm{m}^{2}\right)\end{array}$} & 2.60 & $69.55 \mathrm{~b}$ & $24.54 \mathrm{~b}$ & $25.92 \mathrm{a}$ & $19.18 \mathrm{a}$ \\
\hline & 3.25 & $82.17 \mathrm{a}$ & 30.78 a & $29.86 \mathrm{a}$ & $21.74 \mathrm{a}$ \\
\hline & 3.90 & $83.24 \mathrm{a}$ & $34.01 \mathrm{a}$ & $29.86 \mathrm{a}$ & $19.87 \mathrm{a}$ \\
\hline \multicolumn{2}{|c|}{$\begin{array}{l}\text { Interacción Tipo de poda } x \\
\text { Densidad }\end{array}$} & ns & ns & ns & ns \\
\hline \multicolumn{6}{|c|}{ Tipo de poda $x$ Densidad (plantas $/ \mathrm{m}^{2}$ ) } \\
\hline \multirow[t]{3}{*}{ Española } & 2.60 & $82.88 \mathrm{~b}$ & $25.03 \mathrm{c}$ & $38.35 \mathrm{a}$ & $19.66 \mathrm{ab}$ \\
\hline & 3.25 & $102.99 \mathrm{a}$ & $33.92 \mathrm{ab}$ & $44.69 \mathrm{a}$ & $24.38 \mathrm{a}$ \\
\hline & 3.90 & 99.70 a & $37.05 \mathrm{a}$ & $40.47 \mathrm{a}$ & $22.67 \mathrm{ab}$ \\
\hline \multirow[t]{3}{*}{ Holandesa } & 2.60 & $56.23 \mathrm{c}$ & $24.05 \mathrm{c}$ & $13.49 \mathrm{~b}$ & $18.69 \mathrm{ab}$ \\
\hline & 3.25 & $61.35 \mathrm{c}$ & $27.63 \mathrm{bc}$ & $15.03 \mathrm{~b}$ & $19.10 \mathrm{ab}$ \\
\hline & 3.90 & $66.79 \mathrm{c}$ & $30.96 \mathrm{abc}$ & $19.26 \mathrm{~b}$ & $17.07 \mathrm{~b}$ \\
\hline
\end{tabular}

Nota. Datos que comparten una misma letra no presentan diferencias significativas ( $p \leq .05)$, según la prueba LSD Fisher (ns: no significativa. Fuente: Elaboración propia, a partir de los datos obtenidos.

Algunas veces, el número total de frutos por metro cuadrado es mayor conforme aumenta el número de tallos por planta (Jovicich, Cantliffe y Hochmuth, 1999; Monge-Pérez, 2016), lo cual también se evidenció en esta investigación, tanto para el caso del número total de frutos como para el número de frutos de primera y de segunda calidad, los cuales fueron superiores con la poda española que con la poda holandesa. Sin embargo, en otras ocasiones se han encontrado resultados contrarios (Cebula, 1995; Dasgan y Abak, 2003), o no se han hallado diferencias para esta variable al utilizar diferente número de tallos por planta (Aranguiz, 2002; Jovicich, Cantliffe y Stoffella, 2004).
En Costa Rica, se evaluó el chile dulce Nathalie en invernadero a 1,67 plantas/m2, y se obtuvo una producción total de entre 47,93 y 65,46 frutos/ m2 (Campos, 2009); en la presente investigación se consiguieron en general valores mayores para esta variable, especialmente en los tratamientos con poda española (entre 82,88 y 102,99 frutos/ $\mathrm{m} 2$ ); esto se debió, entre otras razones, a que en el presente trabajo se utilizaron mayores densidades de siembra.

En un ensayo se evaluaron diferentes densidades de siembra y tipos de poda en un chile dulce tipo cuadrado cultivado en invernadero, y se obtuvieron entre 7.0 y 14.4 frutos totales/m2 (Jovicich, Cantliffe $\mathrm{y}$ 
Hochmuth, 1999); dichos valores de producción son muy bajos en comparación con los resultados en la presente investigación, pero hay que tomar en cuenta que las variedades de chile dulce con frutos de forma cónica producen generalmente más frutos por metro cuadrado que las variedades con frutos de forma cuadrada (J. E. Monge-Pérez, datos sin publicar).

En Turquía, al evaluar dos genotipos de chile dulce, se encontraron diferencias significativas entre ellos para el número total de frutos por metro cuadrado, siendo mayor para el genotipo con frutos de menor peso (320.24 frutos $/ \mathrm{m}^{2}$; peso promedio del fruto de $25.5 \mathrm{~g}$ ) que para el genotipo con frutos de mayor peso (129.79 frutos $/ \mathrm{m}^{2}$; peso promedio del fruto de $61.1 \mathrm{~g}$ ) (Dasgan y Abak, 2003); esto enfatiza la influencia del peso del fruto sobre la variable número de frutos por metro cuadrado, por lo que es conveniente tenerlo en cuenta al comparar datos entre diferentes ensayos.

Con respecto a la producción de frutos comerciales por área en chile dulce, diversos investigadores han informado que esta puede variar entre 14.26 y 60.00 frutos comerciales $/ \mathrm{m}^{2}$ (Aranguiz, 2002; Shaw y Cantliffe, 2002; Jovicich, Cantliffe y Stoffella, 2004; Maboko, Du Plooy y Chiloane, 2012); los resultados obtenidos para esta variable en la presente investigación (entre 37.54 y 78.61 frutos comerciales $/ \mathrm{m}^{2}$ ) se acercan y superan dichos valores.

Generalmente, en chile dulce se producen más frutos comerciales por metro cuadrado conforme aumenta la densidad de siembra (Jovicich, Cantliffe y Stoffella, 2004; Maboko, Du Plooy y Chiloane, 2012), tal y como sucedió también en este caso para los frutos de primera calidad.

En relación con el efecto del tipo de poda sobre la producción de frutos comerciales por área, varios investigadores encontraron diferencias significativas entre la poda a cuatro tallos por planta (20.88 frutos $\left./ \mathrm{m}^{2}\right)$, en comparación con la poda a dos o tres tallos por planta (14.26 y 17.36 frutos $/ \mathrm{m}^{2}$, respectivamente) (Maboko, Du Plooy y Chiloane, 2012), lo cual concuerda con los resultados de la presente investigación en el caso de los frutos de primera y de segunda calidad, dado queel número de frutos por metro cuadrado fue mayor con poda española que con poda holandesa para ambas categorías de calidad; sin embargo, otros investigadores no han encontrado diferencias para esta variable al utilizar diferente número de tallos por planta (Aranguiz, 2002; Jovicich, Cantliffe y Stoffella, 2004).

En otro estudio, al evaluar el genotipo de chile dulce FBM-9 en la EEAFBM, a una densidad de 1.56 plantas $/ \mathrm{m}^{2}$, y al utilizar las mismas categorías de calidad empleadas en el presente trabajo, se consiguieron 45.9 frutos totales $/ \mathrm{m}^{2}$, que incluyó 8.3 frutos $/ \mathrm{m}^{2}$ de primera calidad y 18.8 frutos $/ \mathrm{m}^{2}$ de segunda calidad (Quesada, 2015). Esos resultados son mucho más bajos que los obtenidos en la presente investigación para ese mismo genotipo en el caso de la producción total, y de primera y segunda calidad, probablemente debido a que en este ensayo se utilizaron mayores densidades de siembra.

\section{Conclusiones y recomendaciones}

La densidad de siembra y el tipo de poda no tuvieron efecto estadísticamente significativo sobre la altura de la planta, ni sobre la edad a inicio de cosecha.

Se obtuvieron más frutos por planta en todas las categorías de calidad cuando se utilizó la poda española, en relación con la poda holandesa. El mayor número de frutos totales por planta se alcanzó tanto con la densidad de siembra de 2.60 plantas $/ \mathrm{m}^{2}$ como con 3.25 plantas $/ \mathrm{m}^{2}$, en comparación con 3.90 plantas/ $\mathrm{m}^{2}$. Los tratamientos que presentaron la mayor cantidad de frutos totales por planta fueron la 
poda española, tanto con la densidad de 2.60 plantas $/ \mathrm{m}^{2}$ como con 3.25 plantas $/ \mathrm{m}^{2}$.

Con la poda holandesa se obtuvieron frutos con un mayor peso promedio que con la poda española, aunque su número por planta fue menor. La densidad de siembra no influyó sobre el peso promedio del fruto.

El rendimiento total y comercial de chile dulce fue mayor con poda española que con poda holandesa; esto representa, además, un mejor resultado económico con la poda española, no solo por el considerable rendimiento obtenido, sino también por el menor costo, dado que la labor de aplicar la poda holandesa conlleva un aumento significativo en los costos de mano de obra. También se obtuvo un mayor rendimiento total y comercial con las densidades de siembra de 3.25 plantas $/ \mathrm{m}^{2}$ y 3.90 plantas $/ \mathrm{m}^{2}$, en comparación con 2.60 plantas $/ \mathrm{m}^{2}$.

El número de frutos totales, y de primera $\mathrm{y}$ segunda calidad por metro cuadrado, fue mayor con la poda española que con la poda holandesa. Igualmente, se produjeron más frutos totales y de primera calidad por metro cuadrado con las densidades de 3.25 plantas $/ \mathrm{m} 2$ y 3.90 plantas $/ \mathrm{m}^{2}$ que con 2.60 plantas $/ \mathrm{m}^{2}$.

Dado que los tratamientos de poda española, tanto a una densidad de 3.25 plantas $/ \mathrm{m}^{2}$ como de 3.90 plantas $/ \mathrm{m}^{2}$, fueron los que mostraron el mayor rendimiento comercial, así como el mayor número de frutos totales por metro cuadrado, y que también estuvieron entre los cuales produjeron la mayor cantidad de frutos de primera calidad por metro cuadrado, se concluye que son los tratamientos más recomendables para este genotipo en esta localidad. Sin embargo, dado que no hay diferencias significativas entre ellos, desde el punto de vista económico se recomienda el uso de la poda española a una densidad de 3.25 plantas $/ \mathrm{m}^{2}$, pues a esta densidad de siembra los costos son menores, pues se requiere menos semilla. Se recomienda evaluar densidades de siembra mayores en futuras investigaciones.

\section{Agradecimientos}

Los autores agradecen el financiamiento recibido por parte de la Universidad de Florida, así como de la Universidad de Costa Rica, para la realización de este trabajo. Asimismo, agradecen la colaboración de Jendry Portilla, Cristina Arguedas, Jorge Díaz, Julio Vega y Carlos González en el trabajo de campo, y de Mario Monge en la revisión de la traducción del resumen al idioma inglés.

\section{Referencias}

Aguado, G., Del Castillo, J. A., Uribarri, A., Galdeano, J. S., y Sádaba, S. (marzo-abril de 2007). Pimiento tipo Lamuyo en hidroponía. Navarra Agraria, 33-37. Recuperado de http://www.itga.com/docs/PimientoLamuyoHidroponia.pdf

Aminifard, M. H., Aroiee, H., Ameri, A., y Fatemi, H. (2012). Effect of plant density and nitrogen fertilizer on growth, yield and fruit quality of sweet pepper (Capsicum annuum L.). African Journal of Agricultural Research, 7(6), 859-866.

Aranguiz, M. (2002). Efecto de tres sistemas de poda sobre el rendimiento, calidad y asimilados en dos cultivares de pimiento (Capsicum annuum var. grossum L.) producidos orgánicamente bajo invernadero. Talca, Chile: Escuela de Agronomía, Facultad de Ciencias Agrarias, Universidad de Talca.

Arias, J. P. (12 de marzo de 2013). Primera exportación de chile dulce a EE. UU. generó ingresos por $\$ 690.000$. El Financiero.

Borosic, J., Benko, B., Fabek, S., Novak, B., Dobricevic, N., y Bucan, L. (2012). Agronomic traits of soilless grown bell pepper. Acta Horticulturae, 927, 421-428. 
Campos, M. (2009). Efecto de la inoculación de sustratos con Trichoderma spp. sobre el crecimiento y producción de plantas de chile dulce (Capsicum annuum L.), bajo ambiente protegido. San Carlos, Costa Rica: Sede Regional San Carlos, Instituto Tecnológico de Costa Rica.

Cebula, S. (1995). Optimization of plant and shoot spacing in greenhouse production of sweet pepper.ActaHorticulturae, 412, 321-329.

Cruz-Huerta, N., Sánchez, F., Ortiz, J., y Mendoza, M. C. (2009). Altas densidades con despunte temprano en rendimiento y período de cosecha en chile pimiento. Agricultura Técnica en México, 35(1), 70-77.

Dasgan, H. Y., y Abak, K. (2003). Effects of plant density and number of shoots on yield and fruit characteristics of peppers grown in glasshouses. Turkish Journal of Agriculture \& Forestry, 27, 29-35.

Elizondo-Cabalceta, E., y Monge-Pérez, J. E. (2016). Caracterización morfológica de 12 genotipos de chile dulce (Capsicum annuum L.) cultivados en invernadero en Costa Rica. Tecnología en Marcha, 29(3), 60-72.

Elizondo-Cabalceta, E., y Monge-Pérez, J. E. (2017). Evaluación de calidad y rendimiento de 12 genotipos de chile dulce (Capsicum annuum L.) cultivados bajo invernadero en Costa Rica. Tecnología en Marcha, 30(2), 36-47.

Gamayo, J. D. (1996). El cultivo protegido de pimiento. En A. Namesny, Pimientos (págs. 33-40). España: Ediciones de Horticultura.

Grijalva-Contreras, R. L., Macías-Duarte, R., y Robles-Contreras, F. (2008). Productividad y calidad de variedades y densidades de chile bell pepper bajo condiciones de invernadero en el Noroeste de Sonora. Biotecnia, 10(3), 3-10.

Grijalva-Contreras, R. L., Macías-Duarte, R., Robles-Contreras, F., y Valenzuela-Ruiz, M.
J. (2006). Productivity and fruit quality of bell pepper under greenhouse conditions in Northwest Mexico. Hort Science, 41(4), 1075.

Hutton, M. G., y Handley, D. T. (2007). Bell pepper cultivar performance under short, variable growing seasons. Hort Technology, 17(1), 136-141.

Iglesias, N., Roma, F., y Pasini, C. (s. f.). Evaluación de la productividad de cultivares de pimiento (Capsicum annuum) en invernadero en el Alto Valle de Río Negro (temporada 2008/09). Instituto Nacional de Tecnología Agropecuaria, Alto Valle, Argentina.

Jovicich, E., Cantliffe, D. J., y Hochmuth, G. J. (1999). Plant density and shoot pruning on yield and quality of a summer greenhouse sweet pepper crop in Northcentral Florida. Proceedings 28th National Agricultural Plastics Congress, 19-22 May (págs. 184-190). Tallahassee, Florida, EE. UU.

Jovicich, E., Cantliffe, D. J., y Stoffella, P. J. (2003). "Spanish" pepper trellis system and high plant density can increase fruit yield, fruit quality, and reduce labor in a hydroponic, passive-ventilated greenhouse. Acta Horticulturae, 614, 255-262.

Jovicich, E., Cantliffe, D. J., y Stoffella, P. J. (2004). Fruit yield and quality of greenhouse-grown bell pepper as influenced by density, container, and trellis system. Hort Technology, 14(4), 507-513.

Jovivich, E., VanSickle, J. J., Cantliffe, D. J., y Stoffella, P. J. (2005). Greenhouse-grown colored peppers: a profitable alternative for vegetable production in Florida? Hort Technology, 15(2), 355-369.

Lorenzo, P., y Castilla, N. (1995). Bell pepper yield response to plant density and radiation in unheated plastic greenhouse. Acta Horticulturae, 412, 330-334. 
Maboko, M. M., Du Plooy, C. P., y Chiloane, S. (2012). Effect of plant population, stem and flower pruning on hydroponically grown sweet pepper in a shadenet structure. African Journal of Agricultural Research, 7(11), 1742-1748.

Macua, J. I., Lahoz, I., Calvillo, S., y Orcaray, L. (2010). Pimientos California y Lamuyo; variedades y colores campaña 2009. Navarra Agraria, (Enero-Febrero), 32-36.

Mahmoud, A. M., y El-Eslamboly, A. A. (2015). Production and evaluation of high yielding sweet pepper hybrids under greenhouse conditions. American-Eurasian Journal of Agricultural \& Environmental Sciences, 15(4), 573-580.

Maniutiu, D., Sima, R., Apahidean, A. S., Apahidean, M., y Ficior, D. (2010). The influence of plant density and shoot pruning on yield of bell pepper cultivated in plastic tunnel. Bulletin UASVM Horticulture, 67(1), 259-263.

Marín, F. (2010). Cuantificación y valoración de estructuras y procesos de producción agrícola bajo ambientes protegidos en Costa Rica: informe final Proyecto Fittacori F-02-08. Recuperado de http://www.mag.go.cr/bibliotecavirtual/a00290.pdf

Marín, F. (s. f.). Situación general de la agricultura protegida en Costa Rica. Recuperado de http://www.mag.go.cr/bibliotecavirtual/ pronap01-ambiente-protegido.pdf

Monge-Pérez, J. E. (2016). Efecto de la poda y la densidad de siembra sobre el rendimiento y calidad del pimiento cuadrado (Capsicum annuum L.) cultivado bajo invernadero en Costa Rica. Tecnología en Marcha, 29(2), 125-136.

Monsalve, O. I., Casilimas, H. A., y Bojacá, C. R. (2011). Evaluación técnica y económica del pepino y el pimentón como alternativas al tomate bajo invernadero. Revista Colombiana de Ciencias Hortícolas, 5(1), 69-82.

Montaño, N. J., y Belisario, H. D. (2012). Comportamiento agronómico de siete cultivares de pimentón (Capsicum annuum L.). Revista Científica UDO Agrícola, 12(1), 32-44.

Moreno, E. C., Mora, R., Sánchez, F., y García-Pérez, V. (2011). Fenología y rendimiento de híbridos de pimiento morrón (Capsicum annuum L.) cultivados en hidroponía. Revista Chapingo Serie Horticultura, 17(edición especial 2), 5-18.

Paunero, I. (2008). Evaluación de cultivares de pimiento 2006/07. San Pedro, Argentina: Ministerio de Agricultura, Ganadería y Pesca.

Quesada, G. (2015). Producción de chile dulce en invernadero bajo diferentes niveles de agotamiento en la humedad del sustrato. Agronomía Costarricense, 39(1), 25-36.

Quipildor, L. (2001). Evaluación de cultivares de pimiento en invernadero en Lules, Tucumán. Horizonte Agroalimentario, 2(3), 18-19.

Reho, A. I. (13 de setiembre de 2010). Rumbo al norte. Productores de Hortalizas. Recuperado de http://www.hortalizas.com/ehortalizas/?storyid $=2545$

Reséndiz-Melgar, R. C., Moreno-Pérez, E. C., Sánchez-Del Castillo, F., Rodríguez-Pérez, J. E., y Peña-Lomelí, A. (2010). Variedades de pimiento morrón manejadas con despunte temprano en dos densidades de población. Revista Chapingo Serie Horticultura, 16(3), 223-229.

Rotondo, R., Mondino, M. C., Ferratto, J. A., Grasso, R., y Longo, A. (2003). Efecto de la poda de conducción, raleo de frutos y densidad de plantación sobre la productividad del cultivo de pimiento (Capsicum annuum L.), bajo invernadero. Horticultura Argenti$n a, 22(53), 5-9$. 
Sakaldas, M., y Kaynas, K. (2010). Biochemical and quality parameters changes of green sweet bell peppers as affected by different postharvest treatments. African Journal of Biotechnology, 9(48), 8174-8181.

Salas, L. (23 de marzo de 2012). Chile dulce se exportó por primera vez a EE. UU. CRHoy. com Noticias 24/7.

Santos, B. M., Salamé-Donoso, T. P., Obregón-Olivas, H. A., Inestroza, J. E., Galeano, R., Sáenz, M. V., Monge-Pérez, J. E., Cuevas, M. G., Torres-Quesada, E. A., y Méndez-Urbaez, C. J. (2011). Evaluation of planting densities and shoot pruning practices for indeterminate bell pepper production in Dominican Republic, Honduras, and Costa Rica. Proceedings of the Florida State Horticultural Society, 24, 191-193.

Seifi, S., Nemati, S. H., Shoor, M., y Abedi, B. (2012). The effect of plant density and shoot pruning on growth and yield of two greenhouse bell pepper cultivars. Journal of Science and Technology of Greenhouse Culture, 3(11), 77-83.

Sharma, V. K., Semwal, C. S., y Uniyal, S. P. (2010). Genetic variability and character association analysis in bell pepper (Capsicum annuum L.). Journal of Horticulture and Forestry, 2(3), 58-65.

Shaw, N. L., y Cantliffe, D. J. (2002). Brightly colored pepper cultivars for greenhouse production in Florida. Proceedings of the Florida State Horticultural Society, 115, 236-241.

Tsegay, D., Tesfaye, B., Mohammed, A., Yirga, H., y Bayleyegn, A. (2013). Effects of harvesting stage and storage duration on posthar- vest quality and shelf life of sweet bell pepper (Capsicum annuum L.) varieties under passive refrigeration system. International Journal for Biotechnology and Molecular Biology Research, 4(7), 98-104.

Vicente-Conesa, F. E., y Sáez-García, M. J. (2004). Comparación de poda a dos guías, a tres guías, aclareo de tallos y cultivo libre en cultivo integrado de pimiento en invernadero. 31 Seminario de Técnicos y Especialistas en Horticultura, Almagro, Ciudad Real, 2001 (págs. 233-237). Almagro, Ciudad Real, España: Ministerio de Agricultura, Alimentación y Medio Ambiente.

Vicente-Conesa, F. E., Condés-Rodríguez, L. F., Sáez-García, M. J., y García-García, A. J. (2005). Valoración de densidades y eliminación de tallos y frutos en cultivo de pimiento tipo California. 34 Seminario de Técnicos y Especialistas en Horticultura, Murcia, 2004 (págs. 293-314). Murcia, España: Ministerio de Agricultura, Alimentación y Medio Ambiente.

Wahb-Allah, M. A. (2013). Responses of some bell-pepper (Capsicum annuum L.) cultivars to salt stress under greenhouse conditions. Journal of Agricultural \& Environmental Sciences of Damanhour University, 12(1), 1-19.

Zúñiga-Estrada, L., Martínez-Hernández, J. d., Baca-Castillo, G. A., Martínez-Garza, A., Tirado-Torres, J. L., y Kohashi-Shibata, J. (2004). Producción de chile pimiento en dos sistemas de riego bajo condiciones hidropónicas. Agrociencia, 38(2), 207-218.

Recibido: 20 de noviembre de 2017

Aceptado: 26 de abril de 2018 